\title{
Acquired tracheoesophageal fistula repair, due to prolonged mechanical ventilation, in patient with double incomplete aortic arch
}

\author{
Davide Zampieri, Alessandro Pangoni, Giuseppe Marulli, Federico Rea
}

\author{
Thoracic Surgery Unit, Department of Cardiologic, Thoracic and Vascular Sciences, University of Padua, Italy
}

\begin{abstract}
We report a case of the repair of an acquired benign tracheoesophageal fistula (TEF) after prolonged mechanical invasive ventilation. Patient had an unknown double incomplete aortic arch determining a vascular ring above trachea and esophagus. External tracheobronchial compression, caused by the vascular ring, increasing the internal tracheoesophageal walls pressure determined by endotracheal and nasogastric tubes favored an early TEF development. The fistula was repaired through an unusual left thoracotomy and vascular ring dissection. TEFs are a heterogeneous group of diseases affecting critically ill patients. Operative closure is necessary to avoid further complications related to this condition. Pre-operative study is mandatory to plan an adequate surgical approach.
\end{abstract}

\section{Case Report}

A 70-year-old female, affected by myasthenia gravis, was admitted to our Unit because of an early-developed tracheoesophageal fistula (TEF). Patient had an acute respiratory failure, due to allergic reaction to quinolone, needing admission to the ICU and endotracheal intubation. During the stay a tracheostomy was positioned due to a difficult weaning from the mechanical ventilator caused by the myasthenia

Corresponding author: Giuseppe Marulli, Thoracic Surgery Unit, Department of Cardiologic, Thoracic and Vascular Sciences, University of Padua, Via Giustiniani 2, 35100 Padova, Italy.

Tel. +39.049 .8212237 - Fax: +39.049 .8212249 .

E-mail: beppemarulli@libero.it

Key words: Aortic arch anomalies; tracheoesophageal fistula.

Contributions: DZ, AP, GM, manuscript drafting; FR, surgical procedure performing.

Conflict of interest: The authors declare no conflict of interest.

Received for publication: 14 June 18

Accepted for publication: 9 October 18

CC Copyright D. Zampieri et al., 2018

Tipografia PI-ME Editrice, Italy

Monaldi Archives for Chest Disease 2018; 88:974

doi: 10.4081/monaldi.2018.974

This article is distributed under the terms of the Creative Commons Attribution Noncommercial License (by-nc 4.0) which permits any noncommercial use, distribution, and reproduction in any medium provided the original author(s) and source are credited. gravis. In the same setting a naso-gastric tube was placed. After four weeks the patient was weaned from the mechanical ventilation, the naso-gastric tube was removed and the rehabilitation program for oral feeding was started. At that point the suspicion of a tracheo-esophageal fistula was raised because of persistent cough, dysphagia and bolus inhalation in the absence of swallowing alteration. The TEF was hence confirmed by tracheoscopy and esophagogastroduodenoscopy, and it was $1.5 \mathrm{~cm}$ in length and $6 \mathrm{~mm}$ in width, and located $3 \mathrm{~cm}$ above the carina in the posterior-lateral wall of the trachea. With the intent to perform a surgical repair, the patient underwent pre-operative thoracic computed tomography (CT) with detection of an unknown double incomplete aortic arch, determining a vascular ring around the trachea and the esophagus, which presented a distention in its proximal part due to chronic compression caused by the vascular ring (Figure 1). The patient also complained of dysphagia during the past year, but it was only attributed to myasthenia gravis. TEF repair was carried out through left posterior-lateral - fourth interspace - thoracotomy. The vascular ring (Figure 2) was opened dissecting both fibrous cord ligaments, resulting from the left aortic arch involution (between the aberrant left subclavian artery and the diverticulum of the descending aorta) and the left ductus (between the main pulmonary artery bifurcation and the diverticulum of descending aorta). The fistulous tract was located just behind the fibrous cord ligaments and their section was necessary to reach and close the defect (Figure 3). TEF repair was performed by single stitches 4/0 polidyoxanone direct suture for the trachea and by longitudinal, two layers, 4/0 polidyoxanone direct suture for esophagus, followed by apposition of intercostal muscular flap.

Patient was discharged on $38^{\text {th }}$ post-operative day after swallowing and respiratory rehabilitation.

\section{Discussion}

Acquired benign TEF usually derives from a complication of intubation due to a cuff-related tracheal injury and has serious consequences on the patient's health status. Because its surgical management can be challenging it has to be reasonably prevented and avoided [1].

Incomplete double aortic arch with atresia of the distal left arch segment is a form that resembles typical double aortic arches. Because the atresia of a portion of the left arch is present, it results in a non-patent fibrous cord, tethering the anterior left arch to a residual diverticulum (Kommerell diverticulum) in the descending aorta [2]. This situation represents a cause of vascular tracheobronchial congenital compression syndrome in adults. Usually it is an anomaly primarily asymptomatic that could be incidentally found during adulthood. Mechanisms causing symptoms are complex; Hardin et al. [3] attributed to both direct compression and secondary weakness of the airway walls. Principles of surgical correction are based on vascular ring surgical decompression.

In our case, TEF was located at the external compression site and the esophageal dilatation in the zone above the vascular ring was an indi- 


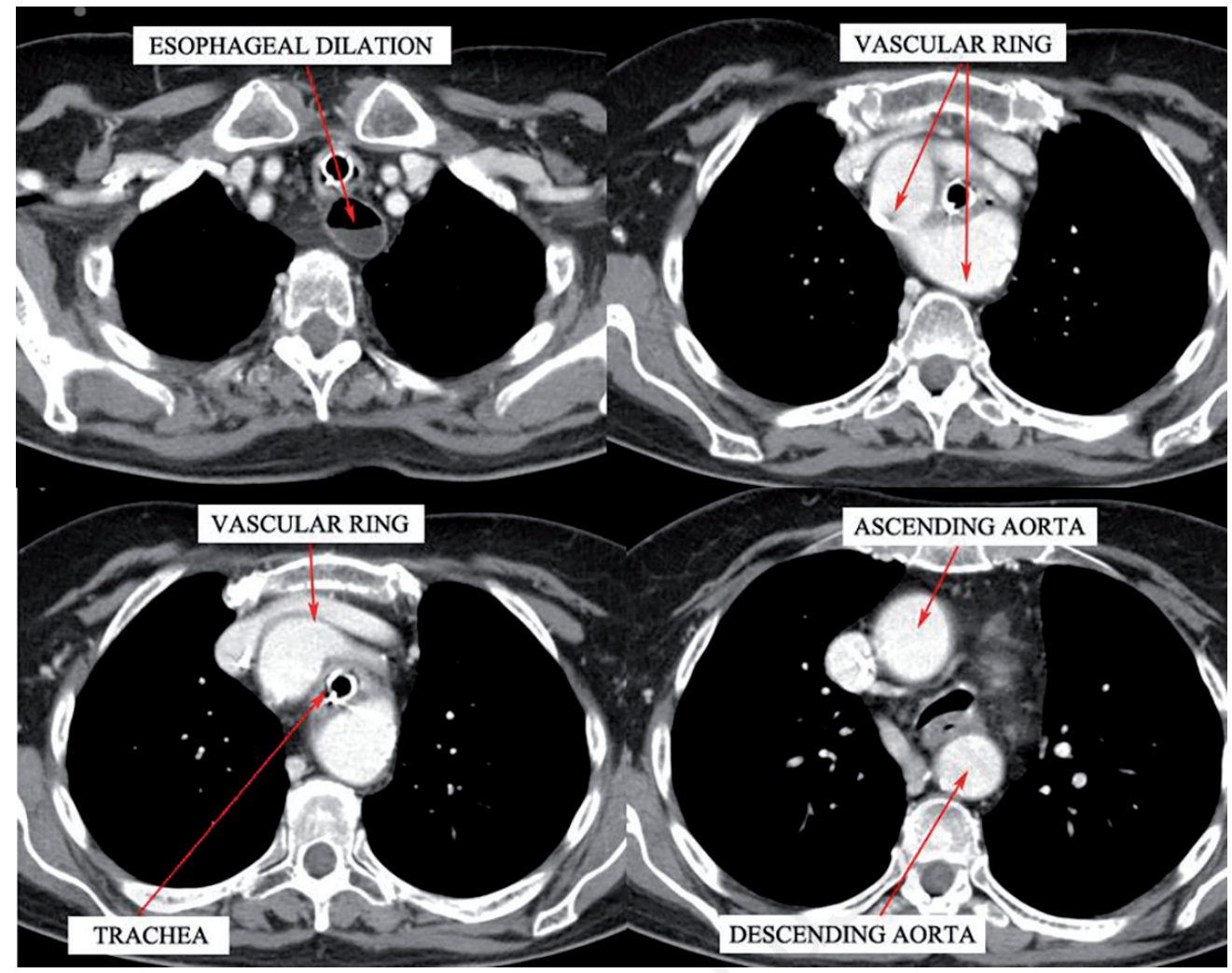

Figure 1. Thoracic CT showing oesophagus dilatation in the zone above the vascular ring compression and the vascular ring.

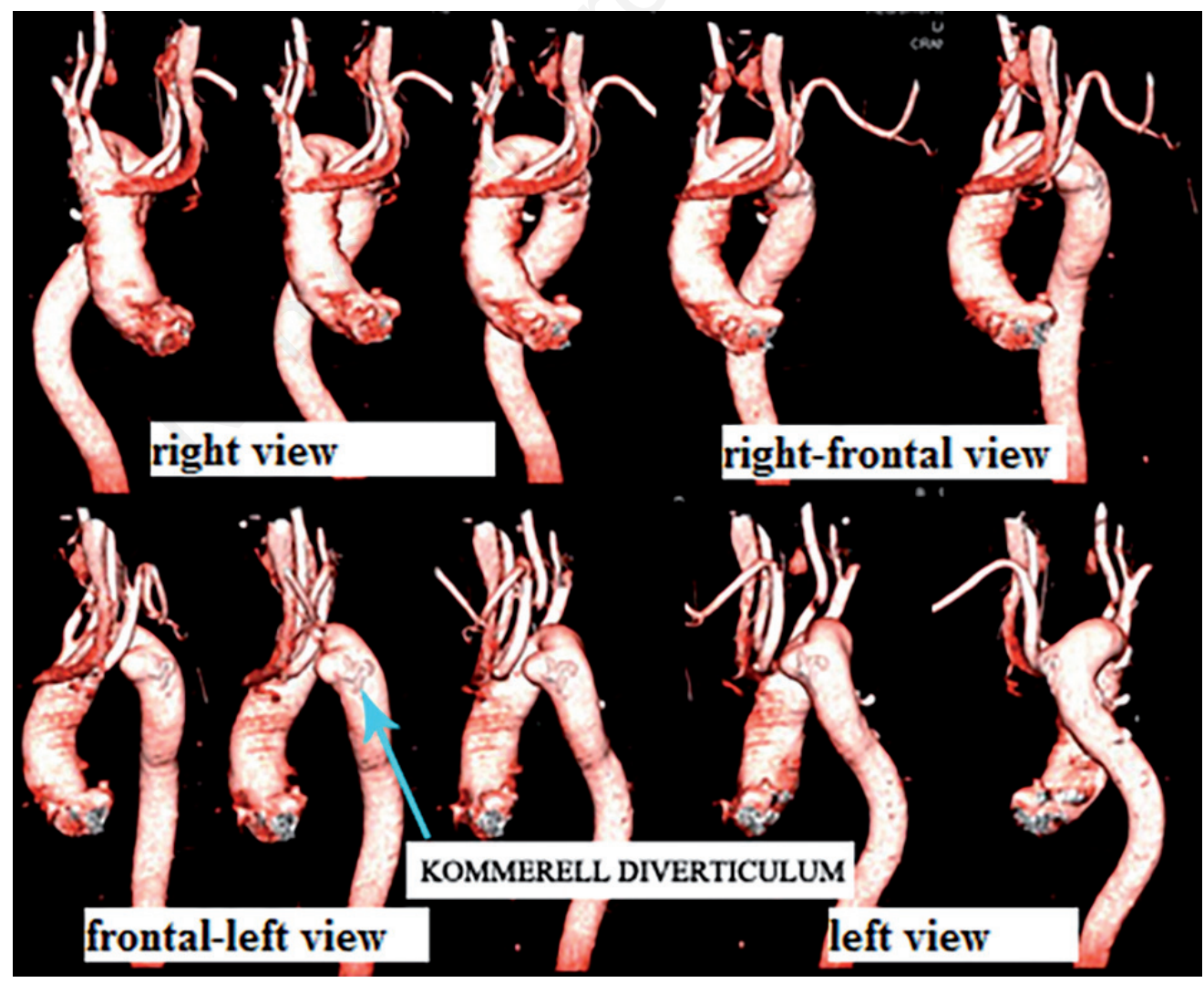

Figure 2. Vascular computed tomography three-dimensional reconstruction of thoracic aorta showing double incomplete aortic arch with atresia of the distal left arch segment and residual diverticulum in the descending aorta. 


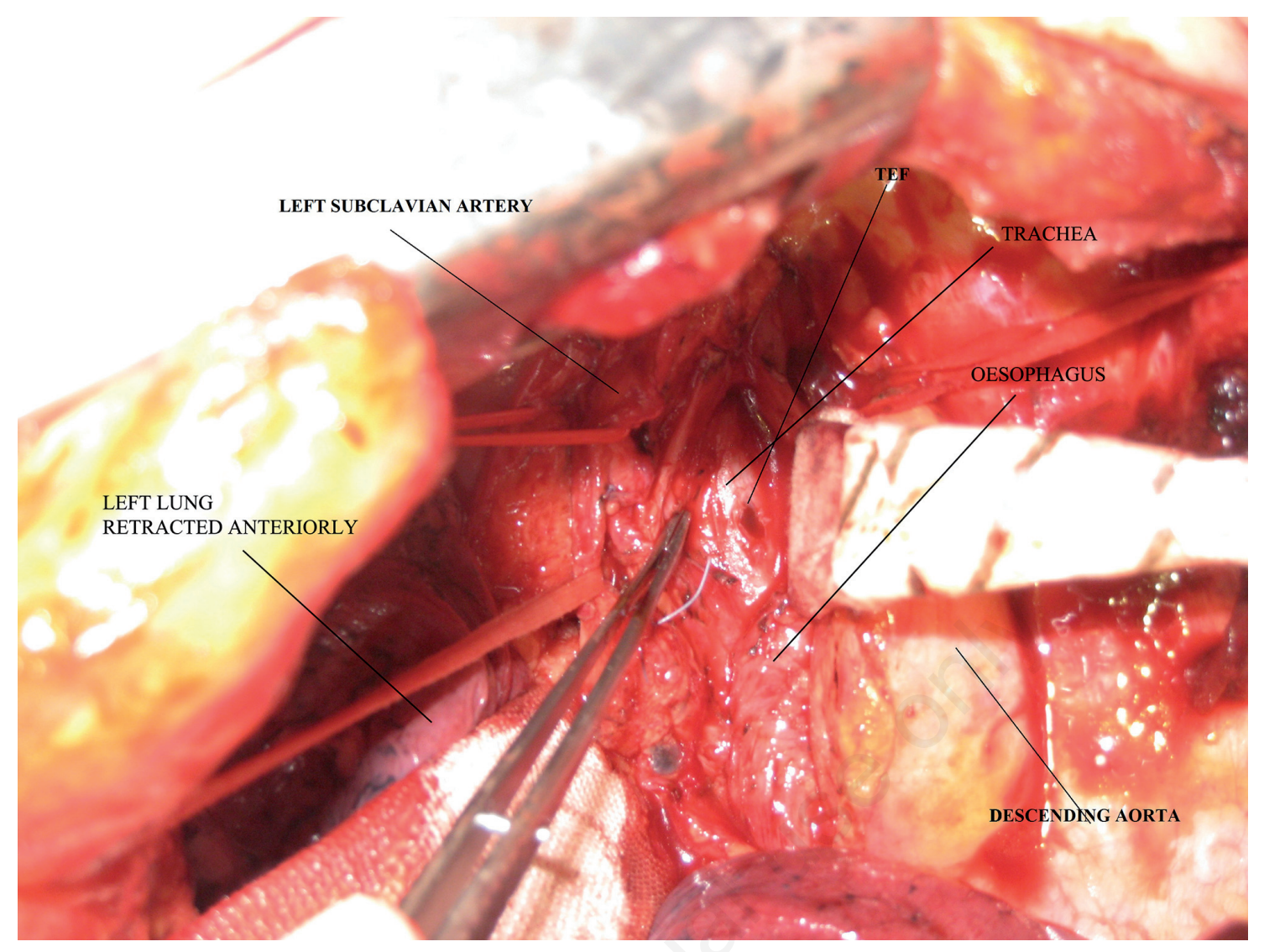

Figure 3. Intraoperative view, trough left thoracotomy, of the tracheoesophageal fistula after vascular ring dissection.

rect sign of compression. The vascular ring external compression on the trachea and esophagus, that were already injured by the presence of the endotracheal and nasogastric tubes, aided TEF development by increasing trans-parietal pressure.

The surgical management of TEF is still controversial and several techniques have been described for its treatment. Surgical approach depends on TEF location: extra-thoracic TEF requires left cervicotomy or anterior collar cervicotomy; intra-thoracic TEF requires right thoracotomy to avoid aorta encumbrance (that would be faced in case of left thoracotomy) [1].

In the reported case, TEF was located intra-thoracically just behind aortic ring. A conventional right thoracotomy approach would have not been appropriate for the presence of double incomplete aortic arch encumbrance that would have hindered TEF closure achievement. The only possible way of access was located between the left aberrant subclavian artery and the diverticulum of the descending aorta, so a left thoracotomy was performed. A recent review dealing with the preoperative utility of thoracic CT in patient affected by TEF and esophageal atresia, concluded that CT helps to identify some of the anatomical factors before surgery but the investigation does not change the surgical approach [4]. In the reported case, in absence of thorax CT images we would have performed an inappropriate right thoracotomic approach. Even if the cited review considers a pediatric population, we can affirm that left thoracotomic approach is necessary in case of TEF surrounded by a double incomplete aortic arch and that CT thorax pre-operative study is mandatory before TEF repair to plan the more appropriate surgical approach.

To our knowledge, this is the first case of acquired TEF associated with double incomplete aortic arch in adulthood; cases of congenital TEF, with esophageal atresia and associated with a vascular ring, have been reported in pediatric surgery [5].

\section{Conclusions}

Here we reported the case of a patient whose treatment has been modified from the standard procedure according to the findings of the pre-operative examination. In conclusion, accurate radiologic chest imaging evaluation and careful planning of the procedure are mandatory in critically ill patients in order to recognize abnormalities that may influence their outcome and treatment.

\section{References}

1. Bibas BJ, Guerreiro Cardoso PF, Minamoto H, et al. Surgical management of benign acquired tracheoesophageal fistulas: A tenyear experience. Ann Thorac Surg 2016;102:1081-7.

2. Schlesinger AE, Krishnamurthy R, Sena LM, et al. Incomplete double aortic arch with atresia of the distal left arch: Distinctive imaging appearance. Am J Roentgenol 2005;184:1634-9.

3. Hardin RE, Brevetti GR, Sanusi M, et al. Treatment of symptomatic vascular rings in the elderly. Tex Heart Inst J 2003;32 411-5.

4. Garge S, Rao KL, Bawa M. The role of preoperative CT scan in patients with tracheoesophageal fistula: a review. J Pediatr Surg. 2013 Sep;48(9):1966-71.

5. Escobar MA, Welke KF, Holland RM et al. Esophageal atresia associated with a rare vascular ring and esophageal duplication diverticulum: A case report and review of the literature. J Pediatr Surg. 2012 0ct:1926-9. 